EPJ Web of Conferences 110,01003 (2016)

DOI: $10.1051 /$ epjconf/201611001003

(C) Owned by the authors, published by EDP Sciences, 2016

\title{
MATHEMATICAL MODELING OF HYDRODYNAMIC AND HEAT TRANSFER IN A TWO-PHASE THERMOSYPHON
}

\author{
Alexander Nee ${ }^{1, a}$ and Atlant Nurpeiis ${ }^{1}$ \\ ${ }^{1}$ National Research Tomsk Polytechnic University, 634050 Tomsk, Russia
}

\begin{abstract}
This paper presents the solution results of conjugate forced convection boundary value problem in a typical rectangular cross-section thermosyphon. The influence of convective heat exchange at the external top horizontal boundary on the heat transfer intensity in such energy removing device is shown. It is found that temperature distributions in thermosyphon steam channel to the $\mathrm{Y}$ direction obtain monotonous character with increasing heat exchange coefficient to the environment.
\end{abstract}

\section{Introduction}

Investigations of jointly proceeding processes of conduction, convection and continuous phase changes in thermosyphon systems of optimum thermal regime control of energy-saturated equipment contribute to a far more efficient designing of such energy removing devices. One of the main disadvantages of the well - known theoretical studies [1-3], which are dealing with the analysis of proceeding thermophysical processes in the thermosyphon, is used of simple heat balance equations. Models [1-3] do not take into account convective heat transfer in the steam channel and conduction in the enclosure elements. Applying an approach [4-6], which is based on the solution of conservation of momentum, mass and energy equations, is of interest.

The aim of this study is numerical simulation of hydrodynamics and heat transfer in a two-phase thermosyphon.

\section{Problem formulation and solution method}

Conductive-convective heat transfer plain problem solved for area, which is shown in figure 1. We consider a typical two-phase rectangular cross-section thermosyphon. The walls of the enclosure are made of copper. Heat flux is supplied to the boundary $\mathrm{y}=$ 0 and $0<\mathrm{x}<$. External vertical walls are considered as adiabatic. Convective heat exchange with the environment are taken into account at the boundary $\mathrm{y}=, 0<\mathrm{x}<$. Equality condition of the temperatures and heat fluxes are accepted on the interfaces "wall liquid", "wall - gas" and "liquid - gas".

The convective effects in the liquid layer are negligible, so the process of heat transfer is carried out in conduction mode. The flow rate of the condensate film is assumed to be constant in time. The vapor is considered as an incompressible viscous heatconducting fluid that satisfies the Boussinesq approximation.

The physical process under study in the forced convection mode is described by the transfer equations of momentum, mass and energy. In variables " vorticity $\omega-$ stream function $\psi-$ temperature $\mathrm{T}$ ” are as follows:

$$
\begin{gathered}
\rho_{1}\left(\frac{\partial \omega}{\partial t}+u \frac{\partial \omega}{\partial x}+v \frac{\partial \omega}{\partial y}\right)=\mu_{1}\left(\frac{\partial^{2} \omega}{\partial x^{2}}+\frac{\partial^{2} \omega}{\partial y^{2}}\right)+\rho_{1} \cdot \beta \cdot g_{y} \frac{\partial T_{1}}{\partial x}, \\
\frac{\partial^{2} \psi}{\partial x^{2}}+\frac{\partial^{2} \psi}{\partial y^{2}}=-\omega \\
\frac{\partial T_{1}}{\partial t}+u_{1} \frac{\partial T_{1}}{\partial x}+v_{1} \frac{\partial T_{1}}{\partial y}=a_{1} \cdot\left(\frac{\partial^{2} T_{1}}{\partial x^{2}}+\frac{\partial^{2} T_{1}}{\partial y^{2}}\right)
\end{gathered}
$$

${ }^{\text {a }}$ Corresponding author: nee_alexander@mail.ru 


$$
\begin{aligned}
& \frac{\partial T_{2}}{\partial t}=a_{2} \cdot\left(\frac{\partial^{2} T_{2}}{\partial x^{2}}+\frac{\partial^{2} T_{2}}{\partial y^{2}}\right), \\
& \frac{\partial T_{3}}{\partial t}=a_{3} \cdot\left(\frac{\partial^{2} T_{3}}{\partial x^{2}}+\frac{\partial^{2} T_{3}}{\partial y^{2}}\right)
\end{aligned}
$$

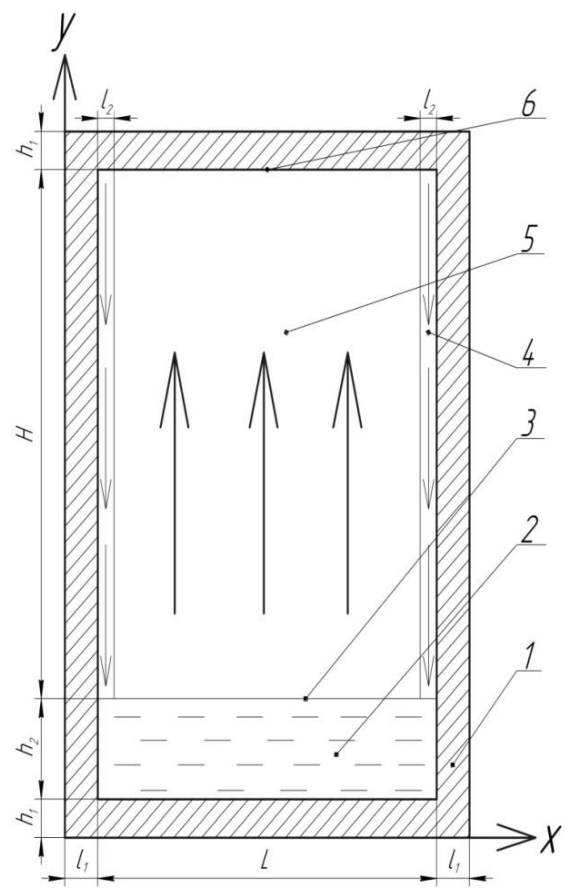

Figure 1. Solution domain: Two-phase thermosyphon: 1 - enclosure; 2 - heat carrier; 3 - evaporation zone; 4 - condensate layer; 5 - steam channel; 6 - condensation zone.

Equations (1) - (5) are modified to the dimensionless form. As the scale distance was selected the steam channel height. To reduce the system of equations to dimensionless form were used the following relationships:

$$
X=\frac{x}{H} ; Y=\frac{y}{H} ; \tau=\frac{t}{t_{0}} ; U=\frac{u}{V_{\text {in }}} ; V=\frac{v}{V_{\text {in }}} ; \Theta=\frac{T-T_{0}}{T_{h}-T_{0}} ; \Psi=\frac{\psi}{\psi_{0}} ; \Omega=\frac{\omega}{\omega_{0}} ; V_{\text {in }}=\sqrt{g \cdot \beta \cdot\left(T_{h}-T_{0}\right) \cdot H} ; \psi_{0}=V_{\text {in }} H ; \omega_{0}=\frac{V_{\text {in }}}{H} ;
$$

The dimensionless vorticity transport, Poisson and energy equations in conditions of forced convection mode in the steam channel and the heat equations for the liquid layer and walls of thermosyphon are as follows [4-6]:

$$
\begin{gathered}
\frac{\partial \Omega}{\partial \tau}+\frac{\partial \Psi}{\partial Y} \frac{\partial \Omega}{\partial X}-\frac{\partial \Psi}{\partial X} \frac{\partial \Omega}{\partial Y}=\frac{1}{\operatorname{Re}} \cdot\left(\frac{\partial^{2} \Omega}{\partial X^{2}}+\frac{\partial^{2} \Omega}{\partial Y^{2}}\right), \\
\frac{\partial^{2} \Psi}{\partial X^{2}}+\frac{\partial^{2} \Psi}{\partial Y^{2}}=-\Omega, \\
\frac{\partial \Theta_{1}}{\partial \tau}+\frac{\partial \Psi}{\partial Y} \frac{\partial \Theta_{1}}{\partial X}-\frac{\partial \Psi}{\partial X} \frac{\partial \Theta_{1}}{\partial Y}=\frac{1}{\operatorname{Re} \cdot \operatorname{Pr}} \cdot\left(\frac{\partial^{2} \Theta_{1}}{\partial X^{2}}+\frac{\partial^{2} \Theta_{1}}{\partial Y^{2}}\right), \\
\frac{\partial \Theta_{2}}{\partial F O_{2}}=\frac{1}{\operatorname{Re} \cdot \operatorname{Pr}} \cdot\left(\frac{\partial^{2} \Theta_{2}}{\partial X^{2}}+\frac{\partial^{2} \Theta_{2}}{\partial Y^{2}}\right), \\
\frac{\partial \Theta_{3}}{\partial F_{3}}=\frac{\partial^{2} \Theta_{3}}{\partial X^{2}}+\frac{\partial^{2} \Theta_{3}}{\partial Y^{2}} .
\end{gathered}
$$


The initial conditions for the equations (6) - (10) are as follows:

$$
\begin{gathered}
\Psi(X, Y, 0)=\Omega(X, Y, 0)=0, \\
\Theta_{1}(X, Y, 0)=\Theta_{2}(X, Y, 0)=\Theta_{3}(X, Y, 0)=0 .
\end{gathered}
$$

Boundary conditions for equations (1) - (5) are as follows:

$$
\begin{aligned}
& X=0,0<Y<\frac{h_{1}+h_{2}+H+h_{1}}{H}: \frac{\partial \Theta_{4}}{\partial X}=0, \\
& X=\frac{l_{1}+L+l_{1}}{H}, 0<Y<\frac{h_{1}+h_{2}+H+h_{1}}{H}: \frac{\partial \Theta_{4}}{\partial X}=0, \\
& Y=0,0<X<\frac{l_{1}+L+l_{1}}{H}: \frac{\partial \Theta_{4}}{\partial Y}=K i, \\
& Y=\frac{h_{1}+h_{2}+H+h_{1}}{H}, 0<X<\frac{l_{1}+L+l_{1}}{H}: \frac{\partial \Theta_{3}}{\partial Y}=B i \cdot\left(\Theta_{3}-\Theta_{e}\right), \\
& Y=\frac{h_{1}}{H}, \frac{l_{1}}{H}<X<\frac{L}{H}:\left\{\begin{array}{l}
\Theta_{3}=\Theta_{2}, \\
\frac{\partial \Theta_{3}}{\partial Y}=\frac{\lambda_{2}}{\lambda_{3}} \cdot \frac{\partial \Theta_{2}}{\partial Y} .
\end{array}\right. \\
& Y=\frac{h_{1}+h_{2}}{H}, \frac{l_{1}}{H}<X<\frac{L}{H}:\left\{\begin{array}{l}
\Theta_{2}=\Theta_{1}, \\
\frac{\partial \Theta_{2}}{\partial Y}=\frac{\lambda_{1}}{\lambda_{2}} \cdot \frac{\partial \Theta_{1}}{\partial Y}-Q_{e} \cdot W_{e} .
\end{array}\right. \\
& Y=\frac{h_{1}+h_{2}+H}{H}, \frac{l_{1}}{H}<X<\frac{L}{H}:\left\{\begin{array}{l}
\Theta_{1}=\Theta_{2}, \\
\frac{\partial \Theta_{1}}{\partial Y}=\frac{\lambda_{2}}{\lambda_{1}} \cdot \frac{\partial \Theta_{2}}{\partial Y}+Q_{c} \cdot W_{c} .
\end{array},\right. \\
& X=\frac{l_{1}}{H}, \frac{h_{1}}{H}<Y<\frac{H}{H}:\left\{\begin{array}{l}
\Theta_{3}=\Theta_{2}, \\
\frac{\partial \Theta_{3}}{\partial X}=\frac{\lambda_{2}}{\lambda_{3}} \cdot \frac{\partial \Theta_{2}}{\partial X},
\end{array}\right. \\
& X=\frac{l_{1}+l_{2}}{H}, \frac{h_{2}}{H}<Y<\frac{H}{H}:\left\{\begin{array}{l}
\Theta_{2}=\Theta_{1}, \\
\frac{\partial \Theta_{2}}{\partial X}=\frac{\lambda_{1}}{\lambda_{2}} \cdot \frac{\partial \Theta_{1}}{\partial X} .
\end{array}\right. \\
& X=\frac{l_{1}+L-l_{2}}{H}, \frac{h_{2}}{H}<Y<\frac{H}{H}:\left\{\begin{array}{l}
\Theta_{1}=\Theta_{2}, \\
\frac{\partial \Theta_{1}}{\partial X}=\frac{\lambda_{2}}{\lambda_{1}} \cdot \frac{\partial \Theta_{2}}{\partial X} .
\end{array}\right. \\
& X=\frac{l_{1}+L}{H}, \frac{h_{1}}{H}<Y<\frac{H}{H}:\left\{\begin{array}{l}
\Theta_{2}=\Theta_{3}, \\
\frac{\partial \Theta_{2}}{\partial X}=\frac{\lambda_{3}}{\lambda_{2}} \cdot \frac{\partial \Theta_{3}}{\partial X} .
\end{array}\right.
\end{aligned}
$$

The flow rate of the condensate film was calculated in the framework of [7]:

$$
V_{3}=\frac{\varphi-\rho \cdot g}{2 \cdot \mu_{3} \cdot V_{0}} \cdot H^{2}\left((X-L)^{2}-l_{2}^{2}\right)-\frac{\tau_{0} \cdot H}{\mu_{3} \cdot V} \cdot\left(l_{2}^{2}+L^{2}-X\right) .
$$

Adopted notation: Fo- Fourier number; Re- Reynolds number; Pr- Prandtl number; Bi- Biot number; $a-$ thermal diffusivity, $m^{2} / \mathrm{s} ; \alpha$ - heat transfer coefficient, Wt $/\left(\mathrm{m}^{2} \cdot \mathrm{K}\right) ; g-$ acceleration of gravity, $\mathrm{m} / \mathrm{s}^{2} ; \mathrm{L}-$ the transverse dimension of the right channel, $\mathrm{m} ; \mathrm{H}$ - the height of the first channel, $\mathrm{m} ; Q_{e}-$ dimensionless analog of the latent heat of evaporation; $Q_{c}-$ dimensionless analog of latent heat of condensation; $W_{e}-$ dimensionless analog of the evaporation rate; $W_{c}-$ dimensionless 
analog of the condensation rate; $\mathrm{T}_{0}$ - thermosyphon temperature at the initial time, $\mathrm{K} ; \mathrm{Th}$ - temperature scale, $\mathrm{K} ; t_{0}-$ time scale, $\mathrm{s}$; $\mathrm{u}, \mathrm{v}$ - the speed of the axes $\mathrm{x}, \mathrm{y}$ respectively, $\mathrm{m} / \mathrm{s} ; \mathrm{U}, \mathrm{V}$ - dimensionless speed corresponding $\mathrm{u}, \mathrm{v} ; V_{\text {in }}-$ velocity scale, $\mathrm{m} / \mathrm{s} ; \mathrm{x}, \mathrm{y}-$ dimensional coordinates, $\mathrm{m} ; \mathrm{X}, \mathrm{Y}$ - dimensionless coordinates, appropriate $\mathrm{x}, \mathrm{y} ; v$ - kinematic viscosity, $\mathrm{m}^{2} / \mathrm{s} ; \lambda-\operatorname{coefficient}$ of thermal conductivity, $\mathrm{Wt} /(\mathrm{m} \cdot \mathrm{K}) ; \tau-$ dimensionless time; $\Theta-$ dimensionless temperature; $\psi-$ stream function, $\mathrm{m}^{2} / \mathrm{s} ; \psi_{0}-$ the scale of the current function, $\mathrm{m}^{2} / \mathrm{s} ; \Psi-$ dimensionless analogue $\psi ; \omega-$ vorticity, $1 / \mathrm{s} ; \omega_{0}-$ scale vorticity, $1 / \mathrm{s} ; \Omega-$ dimensionless analogue $\omega ; \varphi$ - pressure gradient, $\mathrm{Pa} ; \tau_{0}-$ shear stress.

Formulated problem (6) - (10) with the corresponding initial (11), (12) and boundary (13) - (24) conditions was solved by finite difference method as in $[8,9]$. The used algorithm and solution method were tested on a model problem of forced convection in a square cavity with inlet and outlet ports of mass [10]. Stream function value in the center of primary vortex was used as the flow characteristic. The test results are shown in figure 2.

Based on the data analysis, which is presented in figure 2, it can be concluded that obtained results have good agreement with $[10]$ and, consequently, the used algorithm and solution method are able to work.

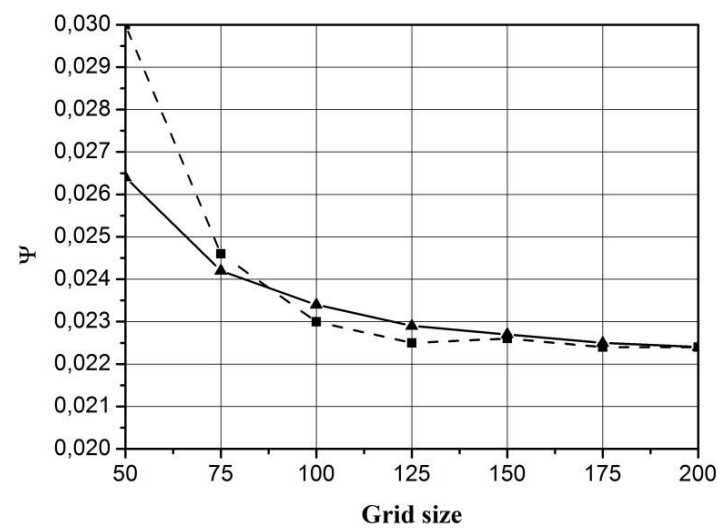

Figure 2. Dependences of the minimum stream functions values in the center of primary vortex versus the computational grid size: $1-[10] ; 2-$ results of this study.

\section{Results and discussion}

Numerical studies were conducted for a typical rectangular cross-section thermosyphon. Distilled water was considered as the heat carrier. The dimensionless complexes were adopted in accordance with the steam flow mode and the geometric characteristics of the heat exchanger under study. Figure 3 shows the fields of temperature and steam velocity at different heat exchange coefficients ( ) at the top of the upper lid. Typical results of formulated boundary value problem are given in dimensional variables for clarity.
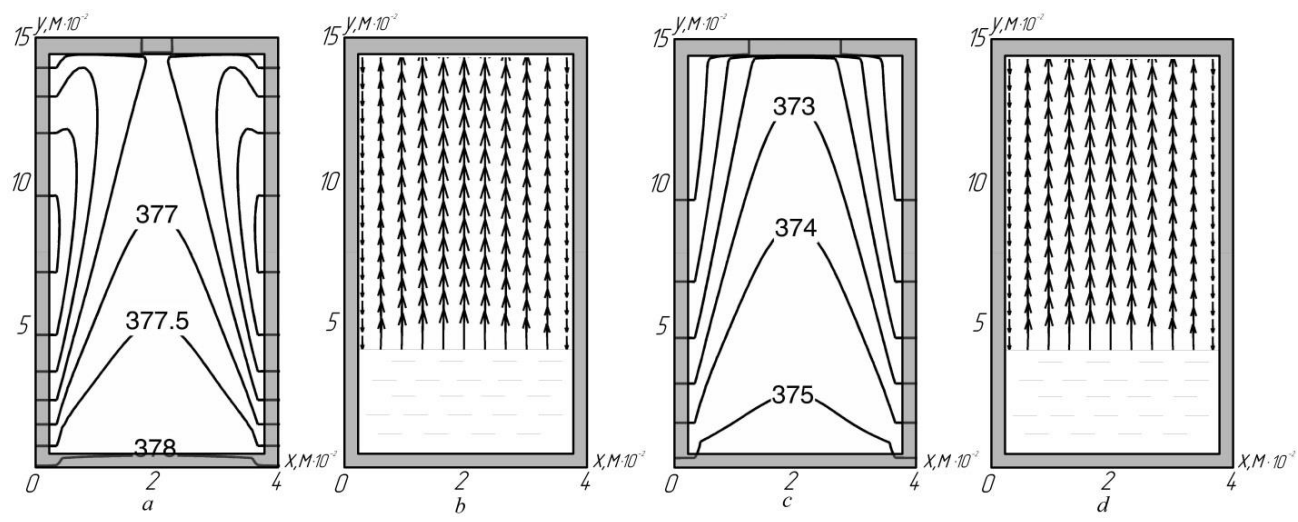


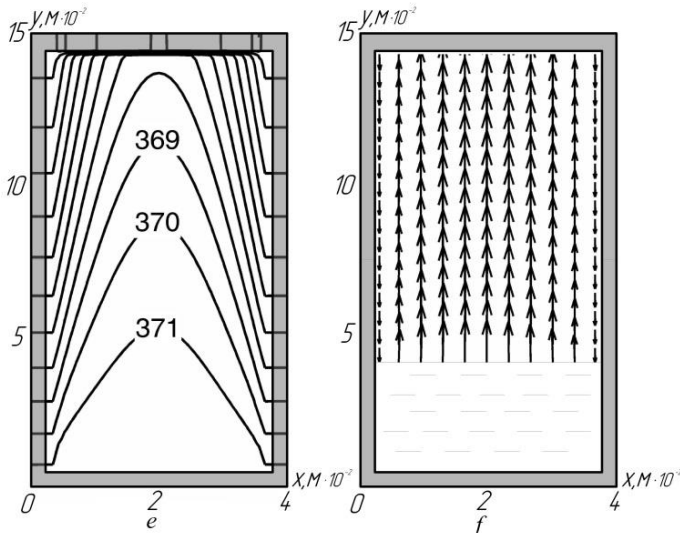

Figure 3. The temperature isolines (a, c, e) and the velocity fields (b, $\mathrm{d}, \mathrm{f})$ at $\operatorname{Re}=250: a, b) B i=0,01 ; c, d) B i=0,03 ; e, f) B i=0.06$.

It is clearly seen that the heat flux, which is coming to the surface $y=0,0<\mathrm{x}<0.04$, initiates the rise in heat carrier temperature, thereby the intensity of the evaporation process is increased. The steam moves toward the $y$-axis to the top horizontal lid. Reaching the surface $y=0.15,0<x<0.04$, the vapor condenses on this boundary A layer of condensate is flowing down along the vertical walls, going back into the zone of intense evaporation.

In order to investigate the impact of convective heat exchange intensity at the upper horizontal lid $(y=0.15,0<x<0.04)$ on the temperature field formation in the steam channel the temperature distributions in the specific sections of the solution domain were analyzed. Dependences $\mathrm{T}=\mathrm{f}(\mathrm{x})$ and $\mathrm{T}=\varphi(\mathrm{y})$ are shown in figure 4 .
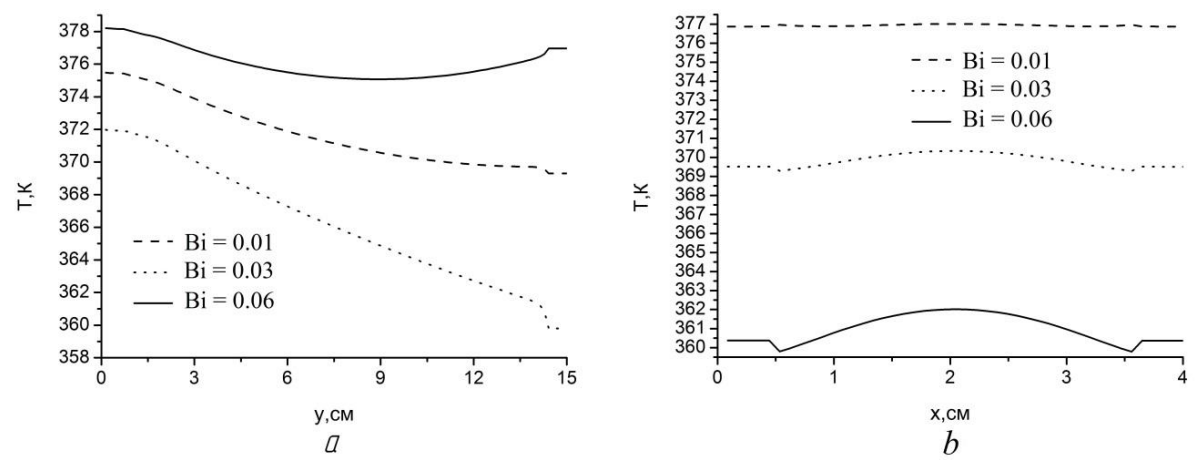

Figure 4. Dependences of temperature distributions versus thermosyphon height: a) $x=0.02$; b) $y=0.15$.

It was found that the heat exchange coefficient increasing at the surface $y=0.15,0<x<0.04$ reduced the temperature in the steam channel. Temperature rising near the top horizontal boundary at $\mathrm{Bi}=0.01$ (fig. 4 a) is explained by the reason that latent heat of evaporation (for distilled water $2300000 \mathrm{~J} / \mathrm{kg}$ ) is released during the condensation process. Dependences $\mathrm{T}=\mathrm{f}(\mathrm{y})$ obtain monotonic character with heat exchange coefficient increasing, what is connected with the intense energy removal at the boundary $\mathrm{y}=0.15,0<\mathrm{x}<0.04$. The thermosyphon top horizontal lid temperature significantly decreased (fig. 4 b).

\section{Conclusion}

According to the results of numerical simulation it was found that variation of energy removal modes at the thermosyphon top horizontal lid contributes for optimization of heat exchanger under consideration. Conjugate conductive-convective heat transfer model allows taking into account differential parameters valid changes at the different heat loads.

\section{Acknowledgments}

The author of this paper is very grateful to his scientific advisor Kuznetsov G.V. for significant help in problem formulation and results discussion.

The work was performed as part of research Goszadaniya "Science” №13.1339.2014 / K (code Federal Target Scientific and Technical Program 2.1410.2014).The reported investigation was supported by grant of State Task "Nauka” №13.1339.2014/K (code of the Federal Target Scientific and Technical Program no. 2.1410.2014). 


\section{EPJ Web of Conferences}

\section{References}

1. S.J. Chen, J.G. Reed, C.L. Tien, Int. J. of Heat and Mass Tr., 27, 9 (1984)

2. A. Niro, G.P. Beretta, ASME HTD, Heat pipes and thermosyph., 221 (1992)

3. H. Farsi, J.L. Miscevic, V. Platel, N. Mazet, Appl. Th. Eng., 23 (2003)

4. G.V Kuznetsov, M.A. Sheremet, T. and A., 16, 1 (2009)

5. G.V. Kuznetsov and M.A. Sheremet, Int. J. of Heat and Mass Tr., 52, 1 - 2 ( 2009)

6. G.V. Kuznetsov, T.A. Nagornova, A.E. Ni, J. of Engin. Ph. and Thermoph.,88, 1 (2015)

7. P. Semenov, Tech. Phys., 14, 7 - 8 (1944)

8. G.V. Kuznetsov, P.A. Strizhak J. of Engin. Thermoph., 17, 3 (2008)

9. G.V. Kuznetsov, T.A. Nagornova, A.E. Ni, J. of Engin. Ph. and Thermoph., 88, 1 (2015)

10. S.M Saedi, J.M. Khodadadi, Int. J. of Heat and Mass Tr., 49 (2006) 